\title{
Skin potential response for stress recognition in simulated urban driving
}

\author{
Pamela Zontone ${ }^{1}$, Antonio Affanni ${ }^{1}$, Alessandro Piras ${ }^{1}$, Roberto Rinaldo ${ }^{1}$ \\ 1 Polytechnic Department of Engineering and Architecture, University of Udine, Via delle Scienze 206, 33100 Udine, Italy
}

\section{ABSTRACT}

In this paper, we address the problem of possible stress conditions arising in car drivers, thus affecting their driving performance. We apply various Machine Learning (ML) algorithms to analyse the stress of subjects while driving in an urban area in two different situations: one with cars, pedestrians and traffic along the course, and the other characterized by the complete absence of any of these possible stress-inducing factors. To evaluate the presence of a stress condition we use two Skin Potential Response (SPR) signals, recorded from each hand of the test subjects, and process them through a Motion Artifact (MA) removal algorithm which reduces the artifacts that might be introduced by the hand movements. We then compute some statistical features starting from the cleaned SPR signal. A binary classification ML algorithm is then fed with these features, giving as an output a label that indicates if a time interval belongs to a stress condition or not. Tests are carried out in a laboratory at the University of Udine, where a car driving simulator with a motorized motion platform has been prearranged. We show that the use of one single SPR signal, along with the application of ML algorithms, enables the detection of possible stress conditions while the subjects are driving, in the traffic and no traffic situations. As expected, we observe that the test individuals are less stressed in the situation without traffic, confirming the effectiveness of the proposed slightly invasive system for detection of stress in drivers.

\section{Section: RESEARCH PAPER}

Keywords: Stress recognition; Electrodermal Activity; Skin Potential Response; Machine Learning; 3D Driving Simulator

Citation: Pamela Zontone, Antonio Affanni, Alessandro Piras, Roberto Rinaldo, Skin potential response for stress recognition in simulated urban driving, Acta IMEKO, vol. 10, no. 4, article 20, December 2021, identifier: IMEKO-ACTA-10 (2021)-04-20

Section Editors: Roberto Montanini, Università di Messina and Alfredo Cigada, Politecnico di Milano, Italy

Received July 23, 2021; In final form December 7, 2021; Published December 2021

Copyright: This is an open-access article distributed under the terms of the Creative Commons Attribution 3.0 License, which permits unrestricted use, distribution, and reproduction in any medium, provided the original author and source are credited.

Corresponding author: Pamela Zontone, e-mail: pamela.zontone@uniud.it

\section{INTRODUCTION}

Paying attention to drivers' mental wellbeing is crucial to improve safety in road traffic. If not properly treated, stress can lead drivers to engage in risky behaviours [1] and therefore car accidents [2]. A danger situation occurs whenever stress is caused by the driving activity itself as happens to professional [3] and regular drivers [4], or by personal issues as highlighted in [5] in case of economic reasons, or any other kind of reason as described in [6]. A Hidden Markov Model (HMM) system to assess the probability of assuming certain behaviours considering the current emotion is developed in [7]. In [8], a survey describing the methods to recognize emotions in drivers is also provided.

The development of stress detection systems follows two main paths [9]. One is based on physiological signals, including Electrodermal Activity (EDA), Electroencephalogram (EEG), Blood volume pulse (BVP), Electromyography (EMG), Skin Temperature (SKT) and Respiration (RESP) [10], [11]. The second one relies on physical manifestations of stress: data describing human behaviour, for example, could be collected by the Global Positioning System [12] and facial expressions [13].

A common approach is to identify a stress condition with the aid of Machine Learning (ML) and Deep Learning (DL) techniques, as in [14]-[16] where the properties of EEG, ECG and EDA signals respectively are exploited for classification purposes. In [17] different kernel configurations for Support Vector Machines (SVMs) are tested, and then applied to electromyographic signals. An automated way to find the optimal kernel has been used in [18], where the kernel for a Deep Multiple Kernel Support Vector Machine (D-MKL-SVM) is selected through a Multiple-Objective Genetic Algorithm (MOGA). The classifier is then used on ECG data. Different physiological measurements, EDA and ECG signals, are combined in [19], where features are automatically extracted from short signal sections and classified by a multimodal Convolutional Neural Network (CNN). 


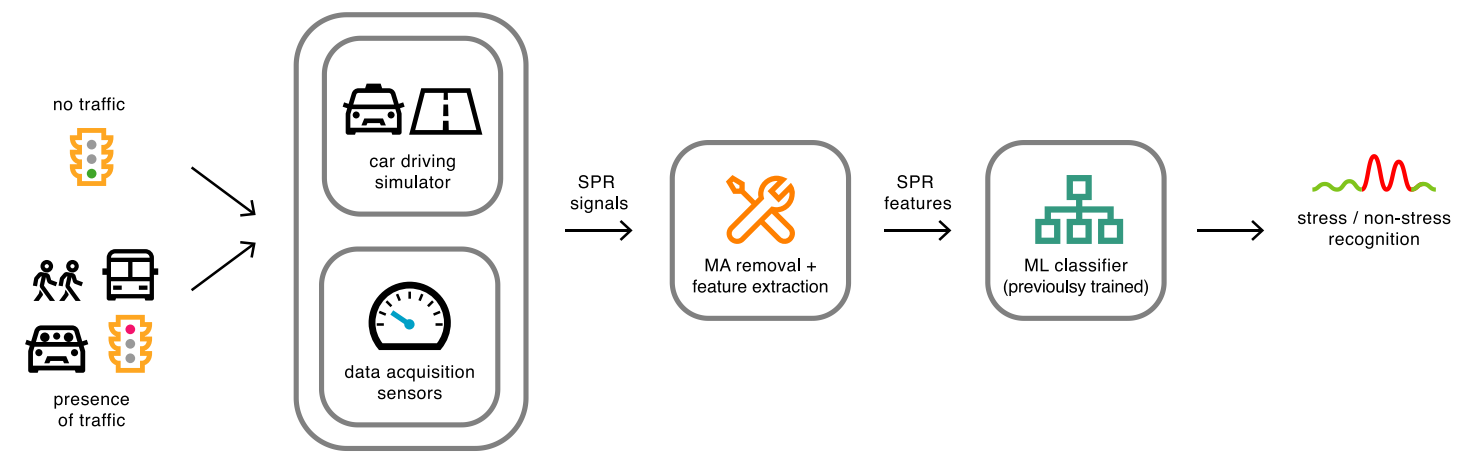

Figure 1. Block scheme of our proposed stress detection measurement system.

A physical approach can be found in [20]. Driver's expressions and eye movements are recorded by near-infrared (NIR) camera sensors, and then aggressive driving behaviour is classified by a CNN. The method proposed in [21] combines both physiological (electrodermal activity) and behavioural (facial) measurements, and fuses together different data types in order to build a Sensor Fusion Emotion Recognition (SFER) system, improving the classification performance.

In previous works, the authors carried out some experiments with the aid of a driving simulator platform, recreating a highway [22], [23] and inducing stress by adding obstacles along the course. We collected the ECG in addition to the Skin Potential Response (SPR) data, and we employed ML and DL algorithms to detect stress in the test individuals. In [24], the authors compared the different physiological responses under manual and autonomous driving tests. In [25], we also examined the possible changes in the physiological responses when different car settings are considered.

One of the main contributions of this paper is the analysis and comparison of the performance results of different ML models (extending the work in [26]), which we demonstrated to be valuable in detecting stress episodes in previous experiments, but now considering the stress caused by urban traffic. In this work, moreover, we simplify the system and consider one signal only, i.e., the SPR signal taken from the hands of the driver. In this way, we propose a slightly invasive setup, which can be arranged with little discomfort for the driver. In detail, we log SPR values from the two hands of individuals while they drive. We apply a Motion Artifact (MA) removal algorithm, to assess the artifacts that can alter the signal caused by the hand movements turning the wheel. This algorithm outputs a single SPR signal, without artifacts, which is fed to an ML classifier, which has been previously trained using a larger dataset. The classifier marks time intervals with a "stress" or "not stress" flag. The individuals were told to drive normally, in an urban setting simulated by the City Car Driving 3D software simulator. The experiment was setup in a way to present two different situations. One situation recreates an urban area with no traffic and empty streets, while the second recreates an urban area complete with traffic, with cars and pedestrians. Findings of this study validate the success of the supervised learning algorithm in its stress detection task. We also demonstrate that SPR signals, recorded with minimally invasive and simple sensors, along with ML classifiers, can detect stress in a reliable way. In the end, we observe that, as expected, stress is generally higher in the urban environment filled with traffic.

The paper is structured as follows. In the next section we present the fundamental blocks of our proposed system. Section 3 introduces the experimental setup. Section 4 discusses the results obtained from our comparative study, where different ML algorithms are used for driver's stress recognition. Finally, some conclusions are drawn in Section 5.

\section{PROPOSED SYSTEM}

The proposed measurement system for stress detection in car drivers is shown in Figure 1.

Each subject under test wears the SPR sensors on the wrists and is seated on the driving simulator available in the BioSens $\mathrm{Lab}$ at the University of Udine. The simulator is composed of a moving platform with two axes (DOF Reality Professional P2), a steering wheel with pedals and gearbox (Logitech G29), and a curved screen. For each subject two different simulations are performed on the same city route with two different conditions: "no traffic" and "traffic". "No traffic" means that there are no other cars nor pedestrians on the road, "traffic" means that we inserted in the simulation other cars and pedestrians with some aggressive events (e.g., lane invasion of other cars or unexpected pedestrian road crossing), as also described in Section 3.

During the entire route planned in the simulations we acquired the SPR signals on the subjects positioning the sensors shown in Figure 2 on the wrist like a smartwatch. The differential voltages from the palm and the back of each hand (VP-VB in Figure 2) are properly conditioned and acquired by a 12 bit A/D converter on board a DSP with sample rate of $200 \mathrm{Sa} / \mathrm{s}$. Data are then sent using a low power WiFi module which operates at $115.2 \mathrm{kbps}$ baud rate. The detailed description of the sensors and their characterization is provided in [27], [28].

Summarizing the architecture and specifications, the sensor analog front end is a band-pass differential amplifier (having input impedance $100 \mathrm{M} \Omega$ ) with maximum input range $\pm 10 \mathrm{mV}$

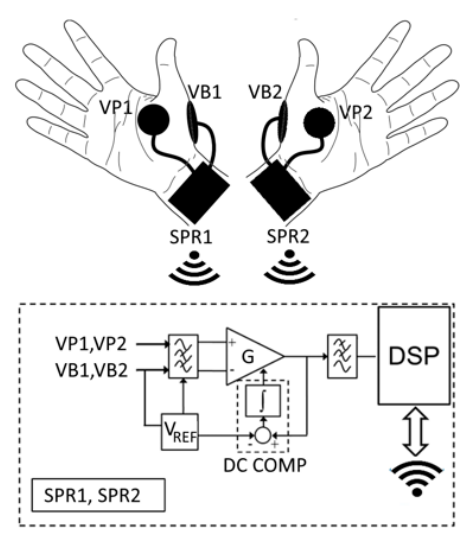

Figure 2. Electrodes arrangement and SPR sensor block diagram. 
and bandwidth in the $[0.08,8] \mathrm{Hz}$ range. The accuracy of SPR acquisition, after characterization, resulted in $0.15 \%$ of full scale (corresponding to $30 \mu \mathrm{V}$ ) and the resolution is $4.9 \mu \mathrm{V}$. The sensors are battery operated with a single LiPo cell with a capacity of $850 \mathrm{mAh}$ ensuring ten hours of transmission, since the current consumption is $85 \mathrm{~mA}$. The sensors form a body network where one SPR sensor acts as slave (henceforth sensor 1) and the other acts as master (henceforth sensor 2). The slave sensor sends packets to the master and the latter aligns the received data packets with the data acquired by the A/D converter. For consumption reasons, the slave can send packets every $40 \mathrm{~ms}$ at minimum. Hence, the slave DSP builds a packet composed of eight data acquired every $5 \mathrm{~ms}$ and sends them to the master. The module is configured as station (STA) with static IP and operates as a UDP client. The gateway address is configured to be the master address.

Figure 3 shows how the packets are built by the DSP on the slave before transmission. The A/D module provides a 12 bit datum every $5 \mathrm{~ms}$. Each byte sent via UART to the master must be identified with a unique code, since the master must recognize if the incoming datum is the upper or lower byte of the slave sample. So, the DSP of the slave builds the lower (upper) byte of information using the six least (most) significant bits of the A/D, adding one bit for lower (upper) byte (L or $\mathrm{H}$ bit in Figure 3, respectively). The data packets received by the master are dismantled and realigned as in Figure 4. The master adds a unique header and builds a packet composed of 18 bytes and containing the information on SPR1 and SPR2. The packet is then sent to a laptop every $40 \mathrm{~ms}$. The data transmitted from the master are acquired by a dedicated Graphical User Interface developed in the .NET environment, and are then processed by a motion artifact (MA) removal algorithm described in [22], [29]. The two SPR signals acquired from the left and right hand of the subjects are processed by the MA algorithm in order to provide as output a single signal that better represents the activity of the Autonomic Nervous System (ANS). As a matter of fact, the SPR signals can be typically affected by motion artifacts due to pressure on the sensors during hand movements. Ideally, the two SPR signals should have approximately the same pattern, since they represent the response to the same stimulus, initiated by the sympathetic response of the ANS. The MA removal algorithm is based on two assumptions, the first being that motion artifact enhances the local energy of the signal. The second being that the motion artifacts rarely appear simultaneously in the SPR signal of both hands. The output of the MA removal block is thus obtained by computing a weighted combination of the two input SPR signals, evaluating their local energy, giving more weight to the less perturbed signal, i.e., the one, between the two input signals, with the least local energy value. In our experiments

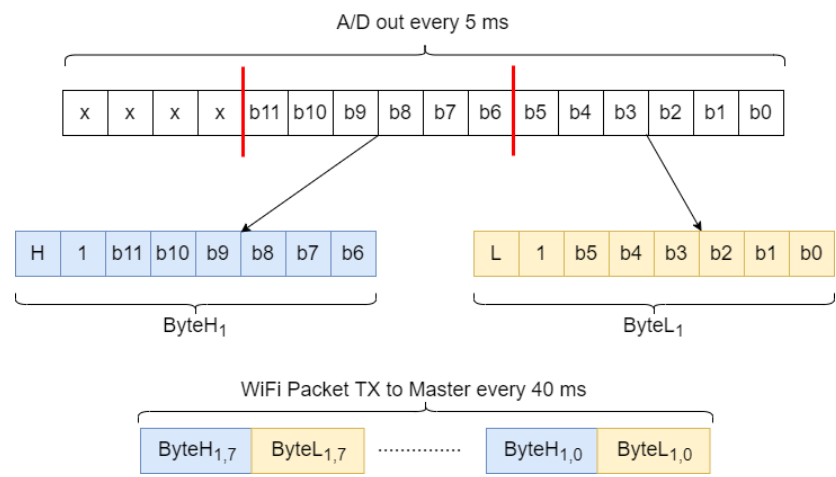

Figure 3. Construction of the packets on the slave for transmission.

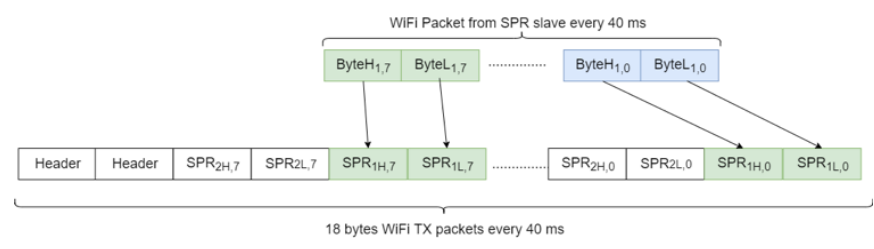

Figure 4. Realignment of the packets on the master.

(see [22]), we found that the motion artifact rarely appears simultaneously in both hands. This, in fact, mostly appears during the steering wheel action, which is predominantly performed by one hand (as also discussed in [24]).

After being processed through the MA removal bock, the cleaned SPR signal is then sent to various ML classification algorithms, which had already been trained on a bigger dataset. This dataset, including 3195 intervals for each stress and nonstress class, is the result of a previous experiment carried out in the VI-grade firm (vi-grade.com), utilizing their professional dynamic simulator. More specifically, in that case, 18 subjects manually drove for $67 \mathrm{~km}$ along a highway, trying to cross 12 obstacles, positioned in prearranged points along the track. These obstacles were: Double lane change (right to left or left to right), Tire labyrinth, Sponsor block (from left or from right), Slalom (from left or from right), Lateral Wind (from left or from right), Jersey LR, Tire trap, Stop. We divided the cleaned SPR signal in $15 \mathrm{~s}$ time interval blocks, after normalization to leverage the signal amplitudes among subjects, and for each block we computed five statistical features: the interval variance, the energy, the mean absolute value, the mean absolute derivative, and the maximum absolute derivative. Each interval overlapped the previous one by $10 \mathrm{~s}$, so we could derive a new feature vector every 5 s. In particular, we could define exactly the obstacle location and span, during which the individuals were supposed to be in a stress state. In this way, we could assign a flag equal to " 1 " to all of the intervals happening to fall or intersect with the stress episodes, and a flag equal to " 0 " to all the others, i.e., the ones happening to fall outside these stress episodes. Finally, after classification of the test set, we applied a re-label step to address the issue related to the number of single and anomalous " 1 " flags [22]. We were able to compare the results of an SVM, a Random Forest (RF) classifier, a Decision Tree (DT), and a k-Nearest Neighbours (k-NN) classifier, which provided a similar accuracy of about $73 \%$, with only the k-NN presenting a slightly lower value $(68 \%)$. All of the ML classifiers were implemented using Matlab (2017.a), and a 10-fold cross validation phase was considered for all of these algorithms. The Bayesian optimization was also used during the training procedure for all of the classifiers (for hyperparameter tuning). A Radial Basis Function (RBF) kernel was employed for the SVM model (see also [23]).

\section{EXPERIMENTAL SETUP}

As already stated, the test was carried out by using a driving simulator, consisting of a 3D driving simulator software and a motorized platform, located in a lab at the University of Udine. The experiment employed 10 test subjects, students of the University of Udine. They were asked to drive along a predefined track, in an urban area simulated by the City Car Driving software. The software enables the creation of an urban area with a nearby motorway, complete of car traffic, with the option of adding different stress-inducing factors, like pedestrian crossing, and vehicles unexpectedly changing lane (also from the opposite direction) or braking suddenly. These stressors do not occur 
Table 1. Total number of intervals marked as "stress" in \%, for each classifier and for each subject in the two driving conditions (with traffic and no traffic). The numeric difference of labels between the two conditions (traffic - no traffic) is also shown.

\begin{tabular}{|c|c|c|c|c|c|c|c|c|c|c|c|}
\hline SVM / Subject & 1 & 2 & 3 & 4 & 5 & 6 & 7 & 8 & 9 & 10 & mean \\
\hline Traffic & 51.82 & 68.00 & 58.86 & 57.47 & 41.94 & 60.00 & 80.33 & 94.49 & 87.60 & 96.43 & 69.69 \\
\hline No traffic & 22.55 & 60.31 & 55.56 & 65.22 & 5.04 & 1.63 & 75.00 & 54.62 & 38.98 & 91.87 & 47.08 \\
\hline Traffic - No traffic & 29.26 & 7.69 & 3.31 & -7.75 & 36.89 & 58.37 & 5.33 & 39.87 & 48.62 & 4.56 & 22.62 \\
\hline RF / Subject & 1 & 2 & 3 & 4 & 5 & 6 & 7 & 8 & 9 & 10 & mean \\
\hline Traffic & 56.93 & 72.00 & 59.49 & 62.07 & 44.35 & 64.44 & 77.05 & 93.70 & 89.26 & 92.14 & 71.14 \\
\hline No traffic & 26.47 & 65.65 & 54.70 & 66.09 & 6.72 & 0.81 & 72.58 & 57.98 & 37.29 & 89.43 & 47.77 \\
\hline Traffic - No traffic & 30.46 & 6.35 & 4.79 & -4.02 & 37.63 & 63.63 & 4.47 & 35.72 & 51.97 & 2.71 & 23.37 \\
\hline DT / Subject & 1 & 2 & 3 & 4 & 5 & 6 & 7 & 8 & 9 & 10 & mean \\
\hline Traffic & 56.20 & 70.40 & 60.76 & 63.22 & 42.74 & 64.44 & 84.43 & 96.06 & 90.91 & 96.43 & 72.56 \\
\hline No traffic & 27.45 & 65.65 & 57.26 & 74.78 & 5.88 & 3.25 & 76.61 & 57.14 & 41.53 & 92.68 & 50.22 \\
\hline Traffic - No traffic & 28.75 & 4.75 & 3.49 & -11.56 & 36.86 & 61.19 & 7.81 & 38.92 & 49.38 & 3.75 & 22.33 \\
\hline k-NN / Subject & 1 & 2 & 3 & 4 & 5 & 6 & 7 & 8 & 9 & 10 & mean \\
\hline Traffic & 56.93 & 70.40 & 58.86 & 63.79 & 42.74 & 63.70 & 75.41 & 92.91 & 87.60 & 90.71 & 70.31 \\
\hline No traffic & 27.45 & 67.94 & 54.70 & 76.52 & 5.04 & 2.44 & 75.00 & 55.46 & 40.68 & 79.67 & 48.49 \\
\hline Traffic - No traffic & 29.48 & 2.46 & 4.16 & -12.73 & 37.70 & 61.26 & 0.41 & 37.45 & 46.93 & 11.04 & 21.82 \\
\hline
\end{tabular}

exactly at the same location and time. However, the type and multiplicity of these stress-inducing events is similar between different simulations. The complete track is displayed in Figure 5. The green solid line represents the motorway, and the orange solid line represents the city route. The subjects were asked to drive in two different situations: in the first there is a complete lack of traffic with no cars and people, whereas the second situation has car and pedestrian traffic. In this second situation, the traffic volume is kind of low, but the behaviour of traffic was set to "very aggressive", where the cars and pedestrians act in a more unpredictable and temperamental way, with cars intruding in the subject's way, or pedestrians crossing the road in forbidden points. One-half of the individuals started with the no traffic situation first, and then proceeded with the traffic situation (i.e., subjects $1,2,3,4$, and 10), while the remaining $50 \%$ did the opposite order (i.e., subjects 5, 6, 7, 8, and 9). Completing the track in Figure 5 takes on average 10 minutes, with a similar required time to complete the motorway and urban section.

\section{EXPERIMENTAL RESULTS}

All of the SPR data collected from the 10 test subjects, after they had driven along a course in an urban area recreated by City Car Driving, are then cleaned through the MA removal block.

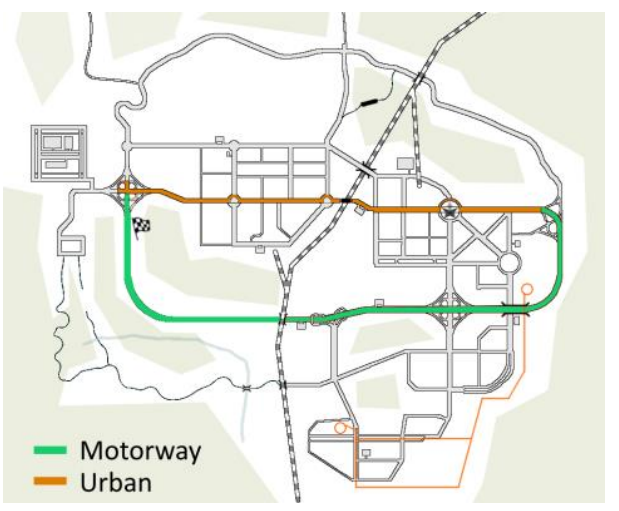

Figure 5. Graphical representation of the course: it comprises a motorway and an urban route.
These output signals are scaled to make a meaningful comparison possible, i.e., for each subject and for each driving condition, we standardize the corresponding signal using the mean and standard deviation resulting from the concatenation of both signals coming from the two driving conditions, with traffic and no traffic, for that subject. These standardized signals are ultimately fed to an ML algorithm. More specifically, the same five SPR features introduced in the previous section are extracted from each $15 \mathrm{~s}$ interval. We make a new interval start 5 seconds after the start of the previous one (therefore each interval is overlapping the previous one by 10 seconds). The various ML classifiers introduced in the previous section are only used for the test phase. In the end, we can look at all of the labels that each classifier gives as output and calculate the final number of labels equal to " 1 " or " 0 ", according to the intervals that it labels as "stress" or "non-stress", for each subject and each driving situation (with and without traffic). Table 1 displays the percentage of labels equal to " 1 " (with stress), when considering the SVM, RF, DT, and k-NN classifiers, for each subject, and taking into account the entire complete test course in the traffic and non-traffic conditions.

As an example, for the RF classifier, we show in Figure 6 the graphical representation of the values reported in Table 1 . As we can deduce looking at all of the classifiers' results, the no traffic situation appears to be less stressful than the traffic situation for all of the subjects excluding subject 4 . In addition, there are some

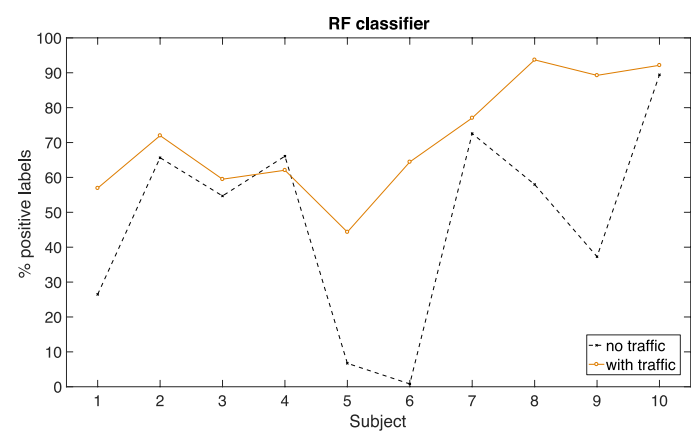

Figure 6. Total number of intervals labelled as "stress" by the RF classifier. 

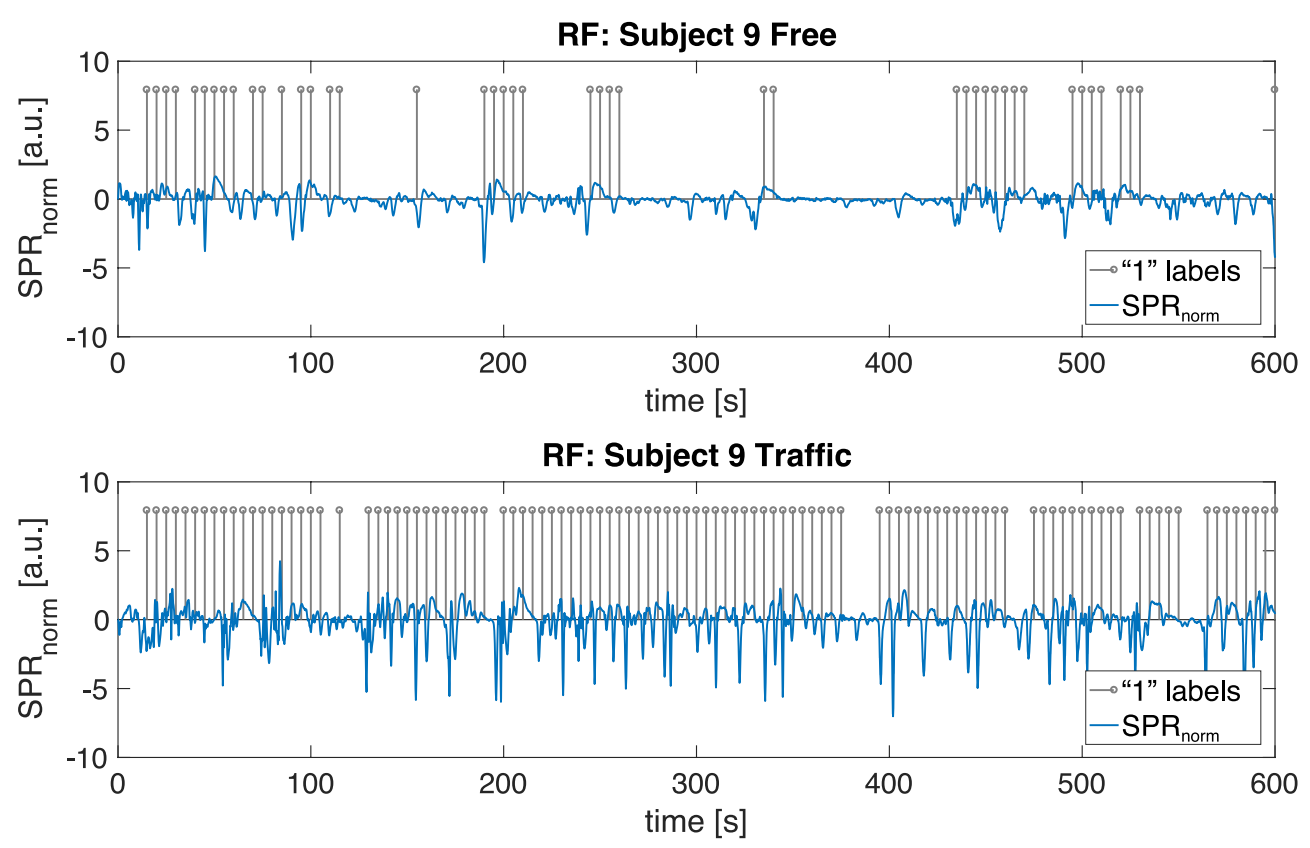

Figure 7. Output of the RF classifier for subject 9 in the two situations (without traffic and with traffic).
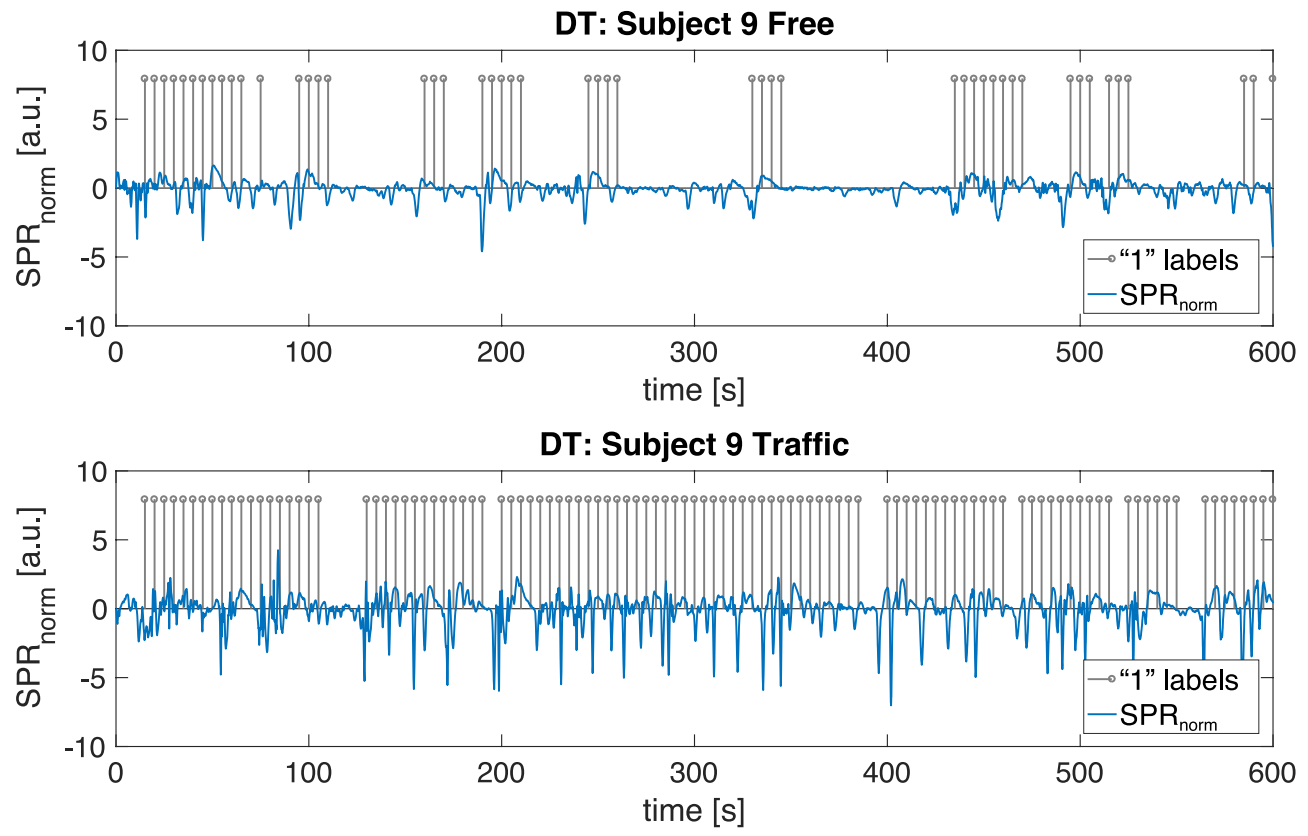

Figure 8. Output of the DT classifier for subject 9 in the two situations (without traffic and with traffic).

individuals where the difference between the positive labels in the two situations is higher (e.g., subjects 5, 6, and 9), while for others this difference is lower (e.g., subjects 3 and 7). We can try to explain this in different ways. Maybe the pressure of taking a test changed the expected stress reaction, or the outcome could be influenced by the order of the simulated situation experienced first by the subjects (traffic and no traffic, or the other way around). Still, for $90 \%$ of the subjects the resulting "stress" interval count is higher in the traffic situation. In Figure 7 we show the output of the RF classifier for subject 9, where the difference between the positive labels in the traffic and no traffic situation is among the biggest positive ones we observe comparing all of the classifiers. For the sake of simplicity, we only plot the positive labels using a grey stem, located at the end of the corresponding $15 \mathrm{~s}$ classified SPR interval. The labels corresponding to the non-stress case are not included in the figure. The cleaned and normalized SPR signals of the subject in the two different situations (with traffic and no traffic) are also shown in a blue continuous line. The output of the DT classifier for the same subject is displayed in Figure 8 (here the difference is slightly lower than the one obtained with the RF). In Figure 9 the output of the k-NN classifier for subject 4 is reported instead. This is the only subject where the difference between the positive labels in the traffic and no traffic scenario is always negative, for all of the classifiers. This negative difference is the biggest for the k-NN case. In Figure 10 we display a last example considering the SVM classifier's output for subject 3. As we can notice, the classifiers well identify the increased stress level throughout the entire simulations. 

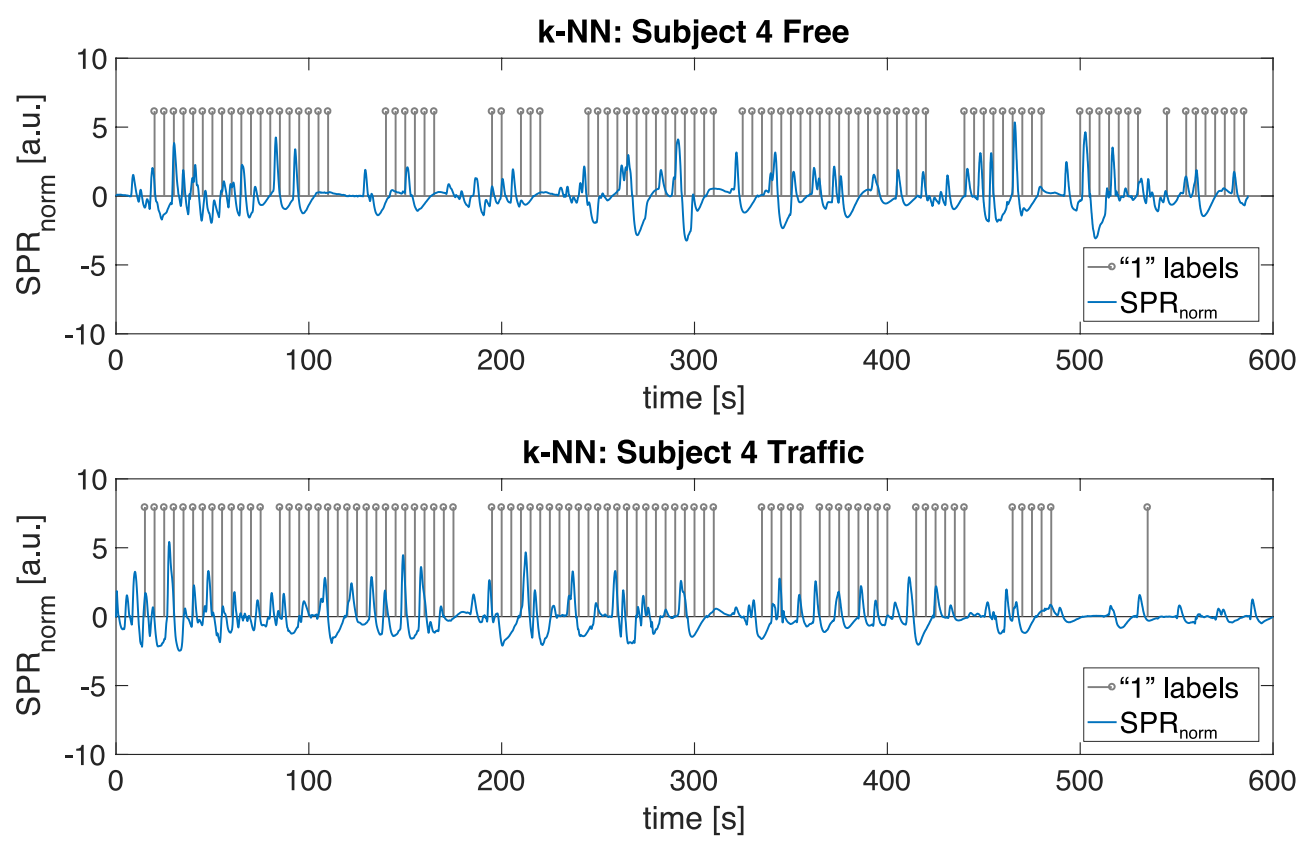

Figure 9. Output of the k-NN classifier for subject 4 in the two situations (without traffic and with traffic).
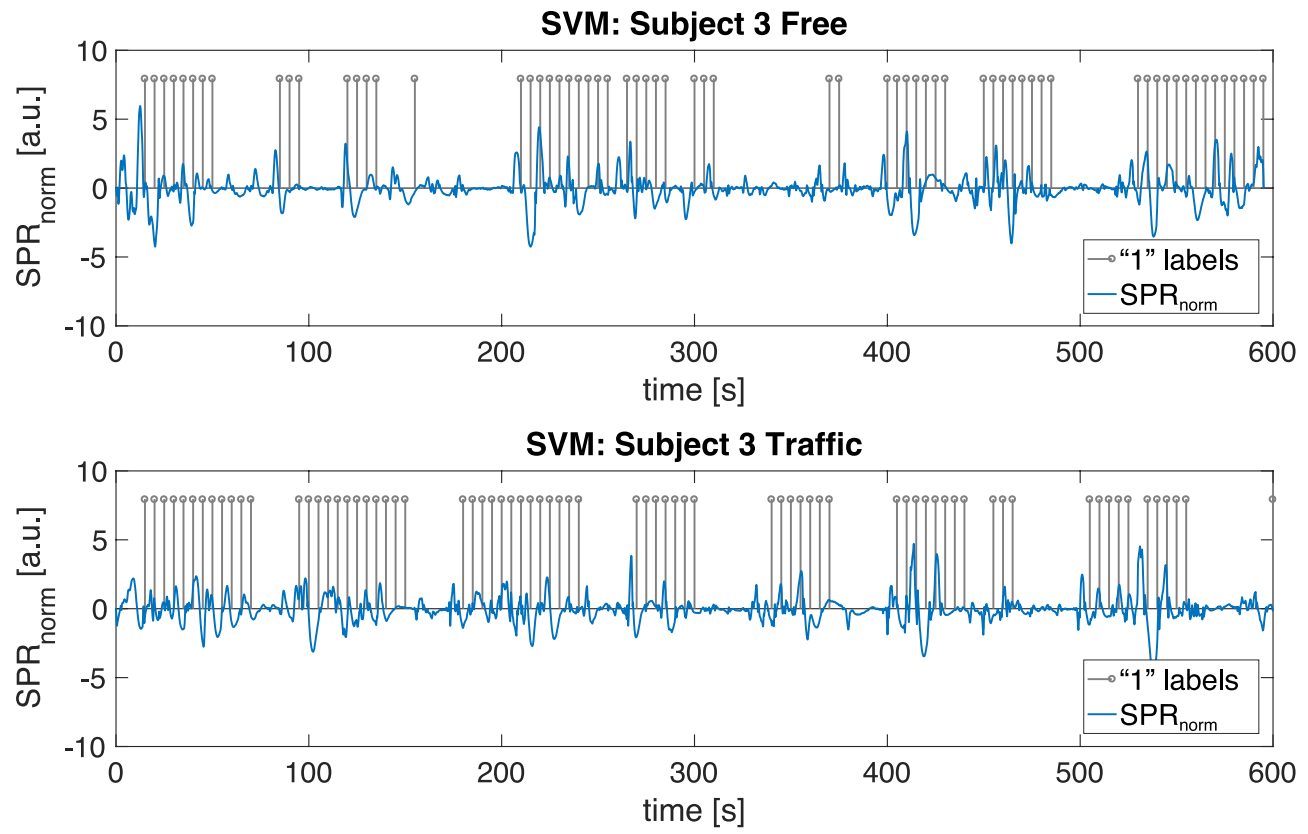

Figure 10. Output of the SVM classifier for subject 3 in the two situations (without traffic and with traffic).

\section{CONCLUSIONS}

In this paper, we described a stress detection system that allows us to identify stress of drivers. Our system classifies overlapping $15 \mathrm{~s}$ signal blocks, and can therefore provide a classification output every $5 \mathrm{~s}$, with a small delay in a real-time application and a good localization in time. The test subjects drove in a simulated urban environment, utilizing a car driving simulator located in our BioSens Lab at the University of Udine. We logged two SPR signals, from their hands, and we processed these signals through an MA removal block. We computed some features from the resulting single signal, and we sent them as input to different ML algorithms, thus comparing the final results. We showed that, regardless of the ML algorithm used, all of the subjects, except one, appeared more stressed when driving in an urban area prearranged with traffic. Therefore, through the use of a low-complexity SPR data acquisition sensor and the application of ML algorithms, we could effectively recognize stress states arising in drivers.

\section{REFERENCES}

[1] L. Bowen, S. L. Budden, A. P. Smith, Factors underpinning unsafe driving: A systematic literature review of car drivers, Transportation Research Part F: Traffic Psychology and Behaviour 72 (2020) pp. 184-210.

DOI: $10.1016 /$ j.trf.2020.04.008

[2] G. Miyama, M. Fukumoto, R. Kamegaya, M. Hitosugi, Risk factors for collisions and near-miss incidents caused by drowsy bus drivers, International Journal of Environmental Research and Public Health 17(12) (2020). DOI: $10.3390 /$ ijerph17124370 
[3] L. R. Hartley, J. El Hassani, Stress, violations and accidents, Applied Ergonomics 25(4) (1994) pp. 221-230. DOI: $10.1016 / 0003-6870(94) 90003-5$

[4] Y.Amichai-Hamburger (edited by), Technology and Psychological Well-being, Cambridge University Press, 2009, Online ISBN 9780511635373

DOI: $10.1017 / \mathrm{CBO} 9780511635373$

[5] D. L. Kitara, O. Karlsson, The effects of economic stress and urbanization on driving behaviours of Boda-boda drivers and accidents in Gulu, Northern Uganda: a qualitative view of drivers, The Pan African Medical Journal 36(47) (2020).

DOI: $10.11604 /$ pami.2020.36.47.21382

[6] E. Bosch, K. Ihme, U. Drewitz, M. Jipp, M. Oehl, Why drivers are frustrated: results from a diary study and focus groups, European Transport Research Review 12(52) (2020) pp. 1-13. DOI: $10.1186 / \mathrm{s} 12544-020-00441-7$

[7] Y. Liu, X. Wang, Differences in driving intention transitions caused by driver's emotion evolutions, International Journal of Environmental Research and Public Health 17(19) (2020). DOI: $10.3390 /$ ijerph17196962

[8] S. Zepf, J. Hernandez, A. Schmitt, W. Minker, R. W. Picard, Driver emotion recognition for intelligent vehicles: a survey, ACM Computing Surveys (CSUR) 53(3) (2020) pp. 1-30.

DOI: $10.1145 / 3388790$

[9] S. Greene, H. Thapliyal, A. Caban-Holt, A survey of affective computing for stress detection: Evaluating technologies in stress detection for better health, IEEE Consumer Electronics Magazine 5(4) (2016) pp. 44-56.

DOI: 10.1109/MCE.2016.2590178

[10] M. Moghimi, R. Stone, P. Rotshtein, Affective recognition in dynamic and interactive virtual environments, IEEE Transactions on Affective Computing 11(1) (2020), pp. 45-62. DOI: $10.1109 /$ TAFFC. 2017.2764896

[11] C. Maaoui, A. Pruski, F. Abdat, Emotion recognition for humanmachine communication, Proc. of the 2008 IEEE/RSJ International Conference on Intelligent Robots and Systems (IROS), Nice, France, 22-26 September 2008, pp. 1210-1215. DOI: $10.1109 /$ IROS.2008.4650870

[12] J. Li, J. Lv, B. Oh, Z. Lin, Y. J. Yu, Identification of stress state for drivers under different GPS navigation modes, IEEE Access 8 (2020) pp. 102773-102783.

DOI: $10.1109 /$ ACCESS.2020.2998156

[13] Su-Jing Wang, Wen-Jing Yan, Xiaobai Li, Guoying Zhao, ChunGuang Zhou, Xiaolan Fu, Minghao Yang, Jianhua Tao, Microexpression recognition using color spaces, IEEE Transactions on Image Processing 24 (12) (2015), pp. 6034-6047. DOI: $10.1109 /$ TIP. 2015.2496314

[14] Hanna Becker, Julien Fleureau, Philippe Guillotel, Fabrice Wendling, Isabelle Merlet, Laurent Albera, Emotion recognition based on high-resolution EEG recordings and reconstructed brain sources, IEEE Transactions on Affective Computing 11(2) (2017), pp. 244-257.

DOI: $10.1109 /$ TAFFC. 2017.2768030

[15] Bosun Hwang, Jiwoo You, Thomas Vaessen, Inez Myin-Germeys, Cheolsoo Park, Byoung-Tak Zhang, Deep ECGNet: An optimal deep learning framework for monitoring mental stress using ultra short-term ECG signals, Telemedicine and e-Health 24(10) (2018), pp. 753-772.

DOI: $10.1089 / \mathrm{tmi} .2017 .0250$

[16] F. Al Machot, A. Elmachot, M. Ali, E. Al Machot, K. Kyamakya, A deep-learning model for subject-independent human emotion recognition using electrodermal activity sensors, Sensors 19(7) (2019), art. no. 1659. DOI: $10.3390 / \mathrm{s} 19071659$

[17] O. Vargas-Lopez, C. A. Perez-Ramirez, M. Valtierra-Rodriguez, J. J. Yanez-Borjas, J. P. Amezquita-Sanchez, An explainable machine learning approach based on statistical indexes and SVM for stress detection in automobile drivers using electromyographic signals, Sensors 21(9) (2021), art. no. 3155. DOI: $10.3390 / \mathrm{s} 21093155$

[18] K. T. Chui, M. D. Lytras, R. W. Liu, A generic design of driver drowsiness and stress recognition using MOGA optimized deep MKL-SVM, Sensors 20(5) (2020), art. no. 1474.

DOI: $10.3390 / \mathrm{s} 20051474$

[19] J. Lee, H. Lee, M. Shin, Driving stress detection using multimodal convolutional neural networks with nonlinear representation of short-term physiological signals, Sensors 21(7) (2021), art. no. 2381 DOI: $10.3390 / \mathrm{s} 21072381$

[20] Rizwan Ali Naqvi, Muhammad Arsalan, Abdul Rehman, Ateeq Ur Rehman, Woong-Kee Loh, Anand Paul, Deep learning-based drivers emotion classification system in time series data for remote applications, Remote Sensing 12(3) (2020), art. no. 587. DOI: $10.3390 / \mathrm{rs} 12030587$

[21] Geesung Oh, Junghwan Ryu, Euiseok Jeong, Ji Hyun Yang, Sungwook Hwang, Sangho Lee, Sejoon Lim, DRER: Deep Learning-Based Driver's Real Emotion Recognizer, Sensors 21(6) (2021), art. no. 2166

DOI: $\underline{10.3390 / \mathrm{s} 21062166}$

[22] Pamela Zontone, Antonio Affanni, Riccardo Bernardini, Alessandro Piras, Roberto Rinaldo, Fabio Formaggia, Diego Minen, Michela Minen, Carlo Savorgnan, Car driver's sympathetic reaction detection through electrodermal activity and electrocardiogram measurements, IEEE Transactions on Biomedical Engineering 67(12) (2020) pp. 3413-3424. DOI: 10.1109/TBME.2020.2987168

[23] Pamela Zontone, Antonio Affanni, Riccardo Bernardini, Leonida Del Linz, Alessandro Piras, Roberto Rinaldo, Supervised learning techniques for stress detection in car drivers, Advances in Science, Technology and Engineering Systems Journal 5(6) (2020), pp. 2229.

DOI: $10.25046 / \mathrm{aj} 050603$

[24] Pamela Zontone, Antonio Affanni, Riccardo Bernardini, Leonida Del Linz, Alessandro Piras, Roberto Rinaldo, Stress evaluation in simulated autonomous and manual driving through the analysis of skin potential response and electrocardiogram signals, Sensors 20(9) (2020), art. no. 2494.

DOI: $10.3390 / \mathrm{s} 20092494$

[25] Pamela Zontone, Antonio Affanni, Riccardo Bernardini, Leonida Del Linz, Alessandro Piras, Roberto Rinaldo, Emotional response analysis using electrodermal activity, electrocardiogram and eye tracking signals in drivers with various car setups, Proc. of the 2020 28th European Signal Processing Conference (EUSIPCO), Amsterdam, NL, 18-21 January 2021, pp. 1160-1164. DOI: 10.23919/Eusipco47968.2020.9287446

[26] P. Zontone, A. Affanni, A. Piras, R. Rinaldo, Stress recognition in a simulated city environment using Skin Potential Response (SPR) signals, Proc. of the 2021 IEEE International Workshop on Metrology for Automotive (MetroAutomotive), Bologna, Italy, 12 July 2021, pp. 135-140. DOI: $10.1109 /$ MetroAutomotive50197.2021.9502867

[27] A. Affanni, Dual-channel electrodermal activity and an ECG wearable sensor for measuring mental stress from the hands, Acta IMEKO 8(1) (2019), pp. 56-63. DOI: $10.21014 /$ acta imeko.v811.562

[28] A. Affanni, Wireless sensors system for stress detection by means of ECG and EDA acquisition, Sensors 20(7) (2020), art. no. 2026. DOI: $10.3390 / \mathrm{s} 20072026$

[29] A. Affanni, A. Piras, R. Rinaldo, P. Zontone, Dual channel Electrodermal activity sensor for motion artifact removal in car drivers' stress detection, Proc. of the 2019 IEEE Sensors Applications Symposium (SAS), Sophia Antipolis, France, 11-13 March 2019, pp. 1-6. DOI: $10.1109 /$ SAS.2019.8706023 